\title{
A Memory/Immunology-Based Control Approach with Applications to Multiple Spacecraft Formation Flying
}

\author{
Liguo Weng, ${ }^{1}$ Min Xia, ${ }^{1}$ Kai Hu, ${ }^{1}$ and Zhuhan Qiao ${ }^{2}$ \\ ${ }^{1}$ Nanjing University of Information Science and Technology, Nanjing 210044, China \\ ${ }^{2}$ Nanjing University of Science \& Technology, Nanjing 210094, China \\ Correspondence should be addressed to Liguo Weng; liguoweng@hotmail.com
}

Received 24 December 2012; Accepted 13 April 2013

Academic Editor: Yang Tang

Copyright (c) 2013 Liguo Weng et al. This is an open access article distributed under the Creative Commons Attribution License, which permits unrestricted use, distribution, and reproduction in any medium, provided the original work is properly cited.

\begin{abstract}
This paper addresses the problem of formation control for multiple spacecrafts in Planetary Orbital Environment (POE). Due to the presence of diverse interferences and uncertainties in the outer space, such as the changing spacecraft mass, unavailable space parameters, and varying gravity forces, traditional control methods encounter great difficulties in this area. A new control approach inspired by human memory and immune system is proposed, and this approach is shown to be capable of learning from past control experience and current behavior to improve its performance. It demands much less system dynamic information as compared with traditional controls. Both theoretic analysis and computer simulation verify its effectiveness.
\end{abstract}

\section{Introduction}

Multiple spacecraft formation flying (MSFF) is an emerging concept which represents the idea of distributing the functionality of large spacecraft among smaller, less-expensive, and cooperative spacecrafts [1-5], as conceptually illustrated in Figure 1. The MSFF approach includes several advantages, such as increased instrument resolution, reduced cost, reconfigurability, and overall system robustness. It bears drastic potentials in the application of scientific and especially military purposes. Both NASA and the Air Force have identified spacecraft formation flying as a key technology for the 21st century.

Today, there are dozens of missions either flying, under development, or proposed that use spacecraft flying in formation. For example, Terrestrial Planet Finder (TPF) will look for extrasolar, Earth-like planets [6]; XEUS and the Constellation X-Ray Mission will explore high-energy astrophysical sources with unequaled resolution [7]; both EO-1/L-7 and CloudSat/Picasso-Cera will study the Earth $[8,9]$; the US Air Force's TechSat-21 seeks to push the frontier in microscale MSFF to enable global awareness and rapid access to space.

The MSFF literature can be divided into two main categories based on the ambient dynamic environment. One is in Deep Space (DS), where the relative spacecraft dynamics could be reduced to double integrator form $[1,10]$, and the other is in Planetary Orbital Environment (POE), where spacecrafts are subjected to significant orbital dynamics and environmental disturbances [11-13].

In this paper, a nonlinear MSFF system model which is based on leader-follower architecture around the earth orbit is introduced. Although there has been a number of studies on leader-follower structure, due to the complex orbital environments, traditional control methods encounter great challenges, such as adaptive and variable structure control methods $[5,14,15]$, because under out-space circumstance, it is very hard to get the relative detailed information of the disturbances and uncertainties, even a relatively accurate model for the spacecraft.

In consideration of the deficiencies of traditional control approaches, we propose a memory/immunology-based control approach based on our previous studies [16, 17]. This approach is inspired by human memory and immune systems, and it does not rely on precise information about system model and environment uncertainties. Instead, it makes use of past control experience and current system behavior to generate new control actions, steering the vehicles toward the desired locations/positions. Moreover, this control 


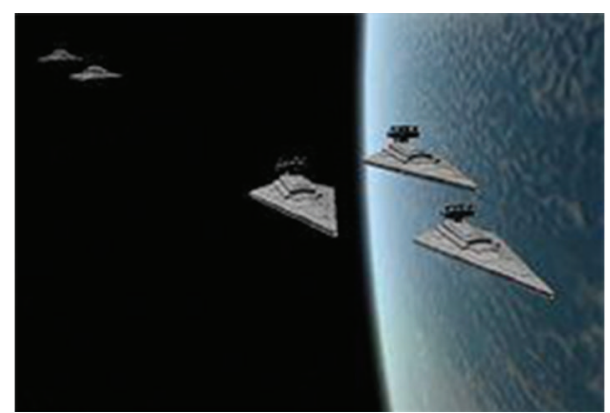

FIGURE 1: Spacecraft formation flying.

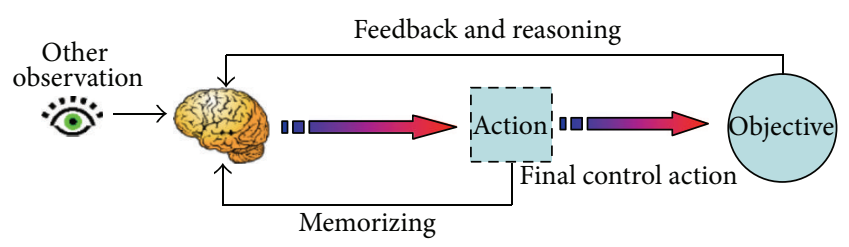

FIGURE 2: Human memory/learning system.

approach demands less computation as compared with most other methods. Both theoretical proof and simulation results confirm the effectiveness of the proposed approach.

\section{Biological Fundamentals}

2.1. Human Memory System. Human memory is one of the most intriguing biological phenomena in the nature; it is what makes possible so many of our complex cognitive functions, including communicating and learning.

There are close links between learning and memory. The existence of memory depends on previous learning, and learning can most clearly be demonstrated by good performance on memory test. Learning and memory involve three stages (see Figure 2):

(1) encoding, involving the processes occurring during presentation of the learning material;

(2) storage, in which, as a result of encoding, some information is stored within the memory system;

(3) retrieval, which involves recovering or extracting stored information from the memory system.

Analogies between the memory system (MS) and the control system (CS) are presented in Table 1.

2.2. Immune System. The protection system that eliminates foreign substances that invade a living body is called the immune system [18].

The human immune system works on two levels with the general goal of pathogen control: a general response mechanism, called innate immunity that does not directly respond to any specific pathogen, and a specific, antibody-mediated response mechanism called acquired immunity.

Each type of B-cell in the immune system is genetically programmed to secrete antibodies that are able to recognize
TABLE 1: Analogies between MS and CS.

\begin{tabular}{ll}
\hline Memory system & Control system \\
\hline Past experience & Past control signal \\
Current (final) response & Current control output \\
Observations & Available sys. info. \\
Feedbacks (comparison) & Control errors \\
Objective & Desired system response \\
\hline
\end{tabular}

TABLE 2: Analogies between IS and CS.

\begin{tabular}{ll}
\hline Immune system & Control system \\
\hline Antigen & System fault \\
Antibody & Control action \\
Stimulated & Proper control action \\
Suppressed & Improper control action \\
\hline
\end{tabular}

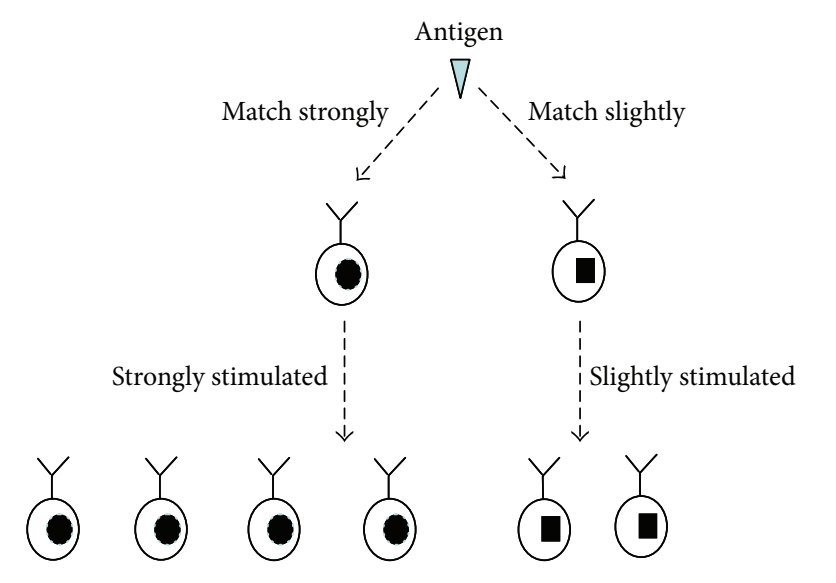

Figure 3: Clone selection.

one particular antigen. Hence, the immune system as a whole can recognize large numbers of antigens; any type of B-cell is just a tiny proportion of the whole.

When one type of antigen is recognized and binds to an antibody, this type of B-cell that secretes such antibodies is induced to proliferate rapidly. In other words, it is stimulated. Hence, more appropriate antibodies are generated to deal with the infection. One type of antigen may be recognized by several types of antibodies, among which the most suitable are stimulated strongly, and the less suitable receive less stimulation (see Figure 3).

Analogies between the immune system (IS) and the control system are presented in Table 2.

\section{System Model}

In this section, we present a nonlinear MSFF system model [11] characterizing the relative position dynamics of follower spacecrafts to a leader spacecraft. In this model, each spacecraft is considered as a point-mass evolving in free space. The leader spacecraft provides a basic reference motion trajectory from which another desired trajectory is identified by the relative distances along $\{x, y, z\}$ axes for the follower 


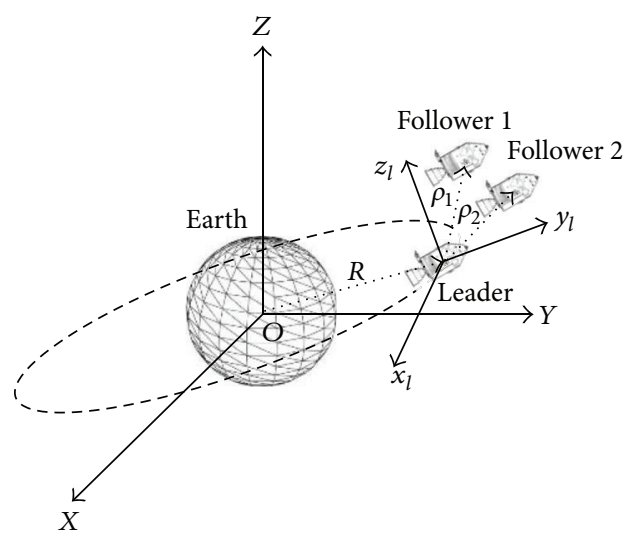

FigurE 4: Schematic representation of the MSFF system.

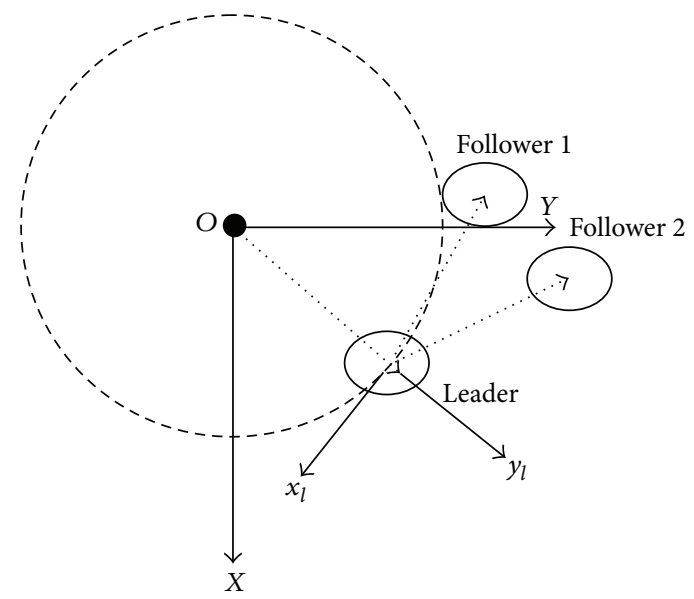

FIgURE 5: Top view of the MSFF system.

spacecraft to track. Furthermore, we assume that the leader spacecraft is in a circular orbit around the Earth. Schematic drawings of the MSFF system are depicted in Figures 4 and 5.

The inertial coordinate system $\{x, y, z\}$ is attached to the center of the earth. Let $R(t) \in \mathbb{R}^{3}$ denote the position vector from origin of the inertial coordinate system to the leader spacecraft and $q_{f}(t) \in \mathbb{R}^{3}$ the position vector from origin of the leader spacecraft coordinate system to the follower spacecraft. The leader spacecraft coordinate system $\left\{x_{l}, y_{l}, z_{l}\right\}$ is attached to the leader spacecraft with the $x_{l}$ axis pointing in the opposite direction as the tangential velocity, the $y_{l}$ axis pointing along the direction of vector $R$, and the $z_{l}$ axis being mutually perpendicular to the $x_{l}$ and $y_{l}$ axes.

The mathematical model governing the dynamic behavior of follower spacecraft relative to the leader spacecraft is

$$
m_{f} \ddot{q}+C(\omega) \dot{q}+N\left(q, \omega, R, u_{l}\right)+\Delta f=u_{f}
$$

or

$$
\begin{gathered}
\ddot{q}=\frac{f(\cdot)}{m_{f}}+\Delta f+\frac{1}{m_{f}} u_{f}, \\
f(\cdot)=-C(\omega) \dot{q}-N\left(q, \omega, R, u_{l}\right),
\end{gathered}
$$

where $u_{f} \in \mathbb{R}^{3}$ is the actual control input vector for the follower spacecraft, $m_{f}$ is the mass of the follower spacecraft, $C(\omega) \in \mathbb{R}^{3 \times 3}$ is the Coriolis-like matrix, $N(\cdot) \in \mathbb{R}^{3 \times 3}$ is a nonlinear term defined by

$$
N(\cdot) \triangleq m_{f}\left[\begin{array}{c}
M G \frac{x}{\|R+q\|^{3}}-\omega^{2} x+\frac{u_{l x}}{m_{l}} \\
M G\left(\frac{y+\|R\|}{\|R+q\|^{3}}-\frac{1}{\|R\|^{2}}\right)-\omega^{2} y+\frac{u_{l y}}{m_{l}} \\
M G \frac{z}{\|R+q\|^{3}}+\frac{u_{l z}}{m_{l}}
\end{array}\right],
$$

with $M$ being the mass of the earth, $G$ being the universal gravity constant, $\omega$ being the angular velocity of the leader spacecraft, and $u_{l x}, u_{l y}$, and $u_{l z}$ being the components of the leader control input vector, and $\Delta f$ is the total disturbance and uncertain forces.

\section{Control Design}

4.1. Inspirations from the Human Memory System. Inspired by the process of the human memory system, the so-called memory-based control method was constructed as follows $[16,17]$ :

$$
u=\omega_{1} U+\frac{\omega_{2} E g^{-1}}{t}
$$

for an arbitrary system expressed as

$$
\dot{x}=f(\cdot)+g(\cdot) u,
$$

where $u$ is the control signal, $U$ stands for the past control experiences, $E$ represents the past feedback information, $\omega_{1}$ is the behavior-memory coefficient vector, and $\omega_{2}$ is the feedback-memory coefficient vector. This structure well resembles the human memory and learning system.

Once stimulated by a given task, the human brain analyzes its surrounding environment (system working environment) and its self-condition (system states) and then integrates those with past experience ( $U$ and $E$ ) to generate the desired control action, which is to be saved in the memory for future retrieval.

More specifically, depending on how many steps (how long) you trace back in your memory, there are different order memory-based controllers. For 1st-order memorybased controllers, where only short-term memory is involved, $\omega_{1}=[1]^{T}, \omega_{2}=[-2,1]^{T}, U=\left[u_{k-1}\right]$, and $E=\left[e_{k}, e_{k-1}\right]$. The subscription $k$ denotes current time $k t$, with $t$ being the sampling period. By using Euler approximation and performing a series of mathematical manipulation for 1storder memory-based control, it is easily shown that as time goes to infinite,

$$
e_{k+1}=t\left(f_{k}-f_{k-1}\right) \text {. }
$$

Therefore,

$$
\left\|s_{k+1}\right\| \leq t^{2} c_{0}
$$


where

$$
c_{0}=\max \left\|\frac{d f}{d t}\right\|,
$$

which denotes the variation rate of the disturbance and uncertainties that cannot be infinite, and the sampling interval $t$ is a very small number, $t$ square is even smaller, and so the tracking error is bound in a very narrow range.

The previous analysis is based on first-order or short-term memory; similar analysis can be made for higher order cases.

However, the problem with the memory-based control is that, at the very beginning, no previous memory was accessible, and, hence, the controller was very immature and showed lots of chattering [17]. Therefore, we turned to the immune system for solutions.

4.2. Inspirations from the Immune System. The human immune system is a dynamic and intelligent system. Once one kind of antigen invades our body, a general immune response is initiated by the immune system. After the invading antigen gets acquainted, a more specific immune response starts to work against the antigen. In other words, because the immune system does not have any knowledge against the antigen, it selects a sort of universal antibody to take effect, and after the antigen is better understood by the intelligent system, a much more matching and effective kind of antibody is activated to fight. Note that the matching antibody could be either already existing or generated from mutation and evolution.

Analogies could be made between the immune system and our control system. The immune system is like a big controller [16, 17], and different kinds of antibodies are components and subcontrollers that work together to achieve a designated objective.

Therefore, a novel approach called memory/immunology-based control was developed. The controller is constructed as follows (Figure 6):

$$
u=(1-\sigma) u_{N}+\sigma u_{A},
$$

where $u$ is the overall control signal (overall immune response), $u_{1}$ and $u_{2}$ are two candidates (two kinds of antibodies in the pool), and $\sigma$ is a function adjusting the concentration of each kind of antibody, which is called immune selection function.

4.2.1. Immune Selection Function. The selection of $u_{N}$ is simple, $u_{N}$ is a general and classic proportional subcontroller, and $u_{A}$ is the memory-based subcontroller. However, how to choose an appropriate immune selection function continues to be a problem. In this paper, an intelligent immune selection function was introduced as follows:

$$
\sigma=1-\exp \left(\frac{-\mu t}{\|e\|+\varepsilon}\right), \quad \mu>0, \varepsilon>0 .
$$

Obviously, this immune selection function incorporates control error. It varies as the magnitude of $e$ fluctuates. When $\|e\|$ is large, which means the antigen is not well understood,

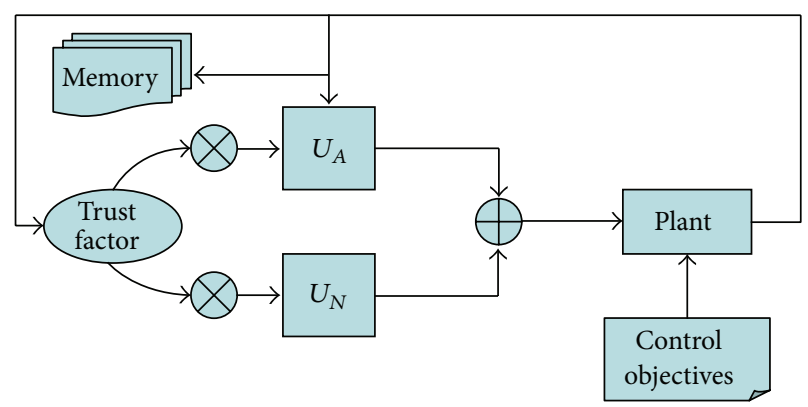

FIGURE 6: Memory/immunology-based control.

the function value approaches 0 , and therefore the general response tends to function more, whereas when $\|e\|$ is small, which means that learned experience has been precise and has been mature, the function value goes to 1 , and thus the specific antibody takes role.

4.3. Control Design for MSFF System. Now we show the detailed memory/immunology-based control design procedure for the MSFF system.

First, we introduce a new variable $s=\dot{e}+\beta e$, where $e=$ $q-q^{*}$ is the tracking error and $\beta$ is an arbitrary positive real number. It is easy to prove that if $s$ goes to zero, $e$ will go to zero too.

The proposed $m$ th-order memory/immunology-based controller is of the form

$$
\begin{gathered}
u=(1-\sigma) u_{N}+\sigma u_{A}, \\
u_{A}=\omega_{1} U+\left(\frac{\omega_{2} m_{f}}{t}\right) s, \\
u_{N}=m_{f}\left(-k s_{k}+\ddot{q}^{*}-\beta \dot{e}\right),
\end{gathered}
$$

with $t$ being the sampling period, $U=\left[u_{k-1}, u_{k-2}, \ldots, u_{k-m}\right]^{T}$ storing the past control experiences, $S=\left[s_{k}, s_{k-1}, \ldots\right.$, $\left.s_{k-m}\right]^{T}$ storing the past feedback information, $\omega_{1} \in \mathbb{R}^{m}$ being the behavior-memory coefficient vector, and $\omega_{2} \in \mathbb{R}^{m+1}$ being the feedback-memory coefficient vector. The $m$ thorder here means that the retroactive effect of the controller is to time $(k-m) t$.

When $m=1$, we have

$$
\begin{gathered}
\omega_{1}=[1]^{T}, \\
\omega_{2}=[-2,1]^{T},
\end{gathered}
$$

and by using Euler approximation and performing a series of mathematical manipulation, it is easily shown that as $T \rightarrow$ $\infty, s_{k+1}=t\left(\Delta f_{k}-\Delta f_{k-1}\right)$. Therefore, $\left\|s_{k+1}\right\| \leq t^{2} c_{0}$, where $c_{0}=\max \|d \Delta f(\cdot) / d t\|$, which denotes the variation rate of the disturbance and uncertainties that cannot be infinite, and the sampling interval $t$ is a very small number, $t$ square is even smaller, and so the tracking error is bound in a very narrow range.

The previous analysis is based on first-order controller; similar analysis can be made for higher order case. Presumably, higher order control leads to better control precision. 


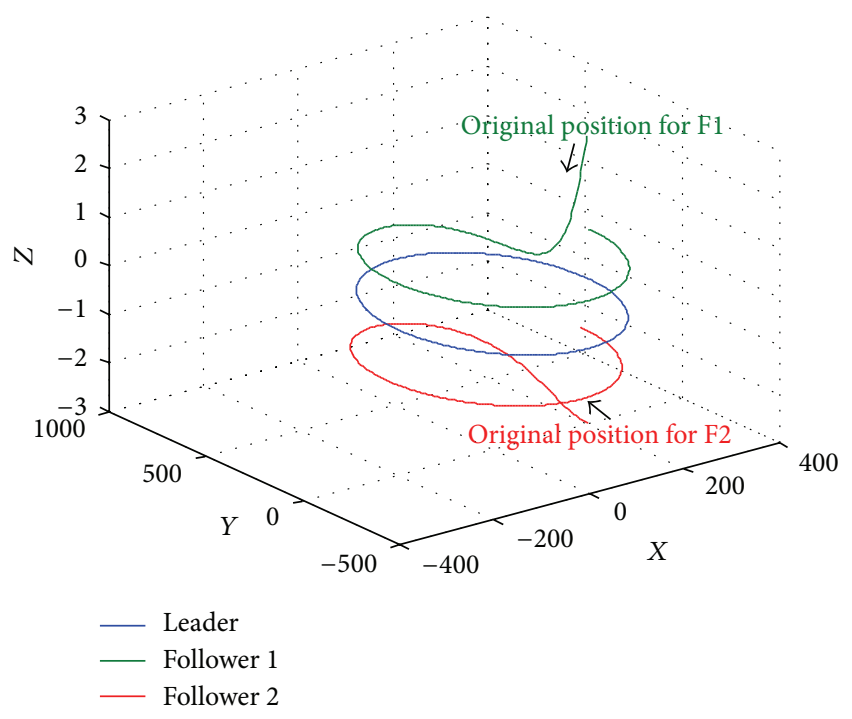

FIGURE 7: Tracking trajectories.

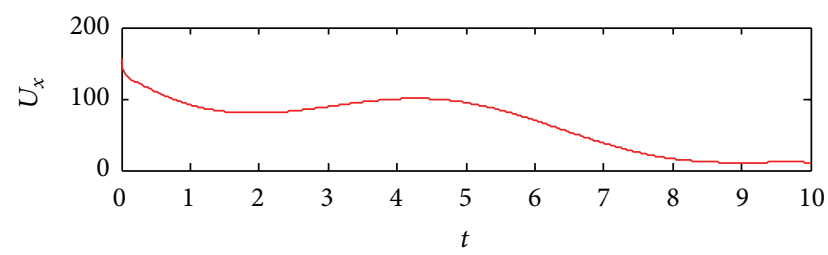

(a)

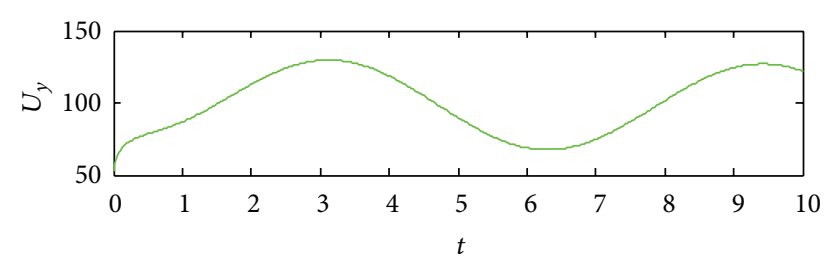

(b)

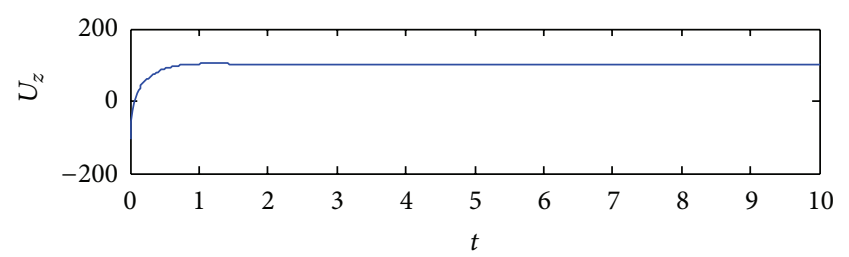

(c)

FIGURE 8: Control forces of follower 1.

\section{Simulation Results}

To verify the effectiveness of the proposed control method, we conduct numerical simulation of three-vehicle formation flight. For simplicity the first-order memory-based controller is applied to the system. The parameters are chosen as:

$$
\begin{aligned}
& t=0.02, \\
& \alpha=0.05,
\end{aligned}
$$

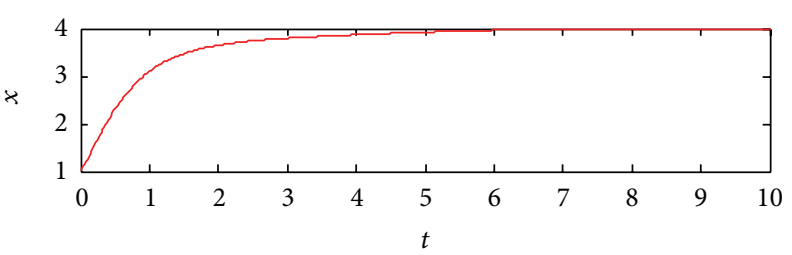

(a)

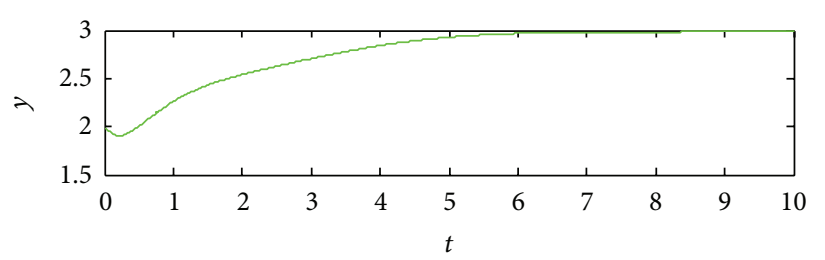

(b)

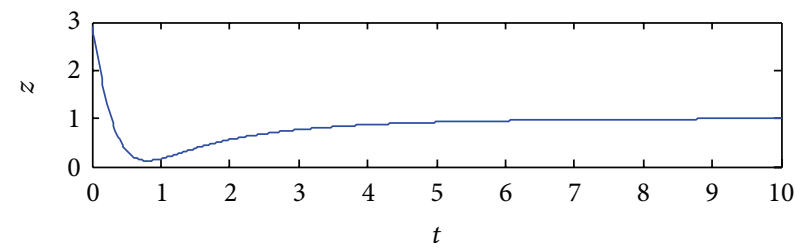

(c)

FIGURE 9: Tracking performance of follower 1.

$$
\begin{gathered}
\gamma=0.02, \\
\beta=5, \\
k=10 .
\end{gathered}
$$

The disturbance is given as

$$
\operatorname{dis}=\left[\begin{array}{c}
20 \sin (T)+2 \cos ^{2}(T) \\
30 \cos (T)+6 \sin ^{2}(T) \\
\sin ^{3}(T)
\end{array}\right] \text {. }
$$

We consider a three-spacecraft MSFF system including one leader and two followers, and our control goal is to keep the three spacecrafts in rigid formation, that is, in another word, to keep the relative position vectors between two followers and the leader constant while the leader is in any arbitrary orbital trajectory.

The desired relative position for follower 1 is $[4,3,1]$, and for follower 2 is $[-10,7,-1]$.

In Figure 7, the tracking trajectories are shown, and the leader is flying in an ellipse orbit.

And in Figure 8, we can see the control forces of follower 1 , which is very smooth and bounded.

Next, in Figure 9, we show the tracking performances of follower 1 along each dimension of $\{x, y, z\}$, and at last, in Figure 10, the tracking errors of follower 1 in each dimension are given, from which we can see that under the control of the proposed method, the vehicles are driven to the destination/position quickly and precisely. 


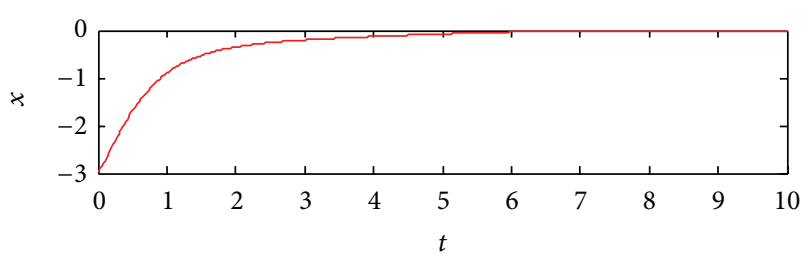

(a)

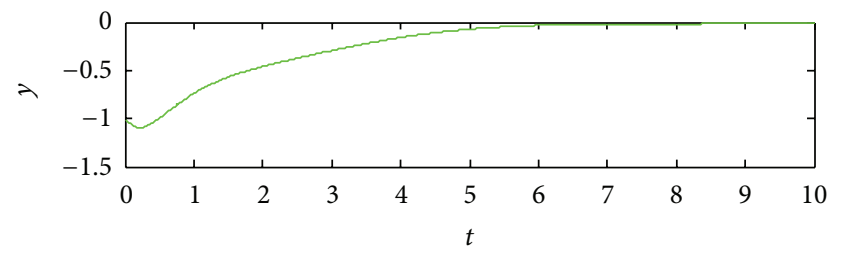

(b)

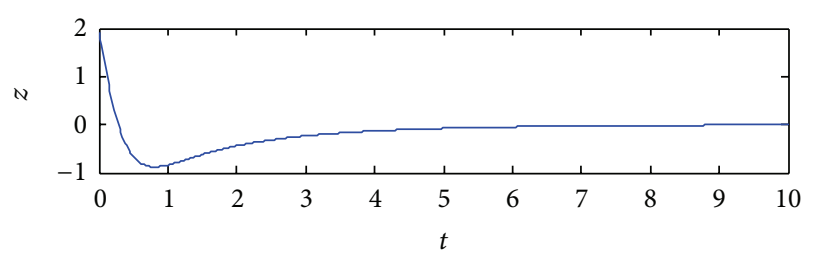

(c)

FIgURE 10: Tracking errors of follower 1.

\section{Conclusion}

A memory/immunology-based concept is used to design a highly robust and adaptive control scheme for close formation flying of multiple spacecrafts. The developed control algorithms are implemented for 3-vehicle system and achieved good performance.

The immunology/memory-based control brings in the inspiration from the immune system. It learns how the immune system generates an appropriate immune response by choosing different antibodies and applying this process into the system controls. This approach is especially efficient and superior when it is applied to a system with lots of disturbances and uncertainties, because of the fact that it requires little information about the disturbances and uncertainties and does not rely on precise model dynamics.

\section{References}

[1] H. Li, Q. Y. Zhang, and N. T. Zhang, "Autonomous navigation of formation flying spacecrafts in deep space exploration and communication by hybrid navigation utilizing neural network filter," Acta Astronautica, vol. 65, no. 7-8, pp. 1028-1031, 2009.

[2] R. Kristiansen and P. J. Nicklasson, "Spacecraft formation flying: a review and new results on state feedback control," Acta Astronautica, vol. 65, no. 11-12, pp. 1537-1552, 2009.

[3] Y. Lv, Q. Hu, G. Ma, and J. Zhou, "6 DOF synchronized control for spacecraft formation flying with input constraint and parameter uncertainties," ISA Transactions, vol. 50, no. 4, pp. 573-580, 2011.

[4] Y. H. Wu, X. B. Cao, Y. J. Xing, P. F. Zheng, and S. J. Zhang, "Relative motion coupled control for formation flying spacecraft via convex optimization," Aerospace Science and Technology, vol. 14, no. 6, pp. 415-428, 2010.

[5] J. Bae and Y. Kim, "Adaptive controller design for spacecraft formation flying using sliding mode controller and neural networks," Journal of the Franklin Institute, vol. 349, no. 2, pp. 578-603, 2012.

[6] P. R. Lawson, "The terrestrial planet finder," in Proceedings of the IEEE Aerospace Conference, vol. 4, pp. 2005-2011, Big Sky, Mont, USA, March 2001.

[7] B. Battrick and XEUS Steering Committee, X-Ray Evolving Universe Spectroscopy - The XEUS Mission Summary, ESA Publications Division, 2000.

[8] D. Folla and A. Hawkins, "Results of NASA's first autonomous formation flying experiment: earth observing-1 (EO-I)," in Proceedings of the AIAA Guidance Navigation and Control Conference, 2002.

[9] D. Keenan, "A formation flying strategy for CloudSa/WicassoCena," in Proceedings of the IEEE Aerospace Conference, vol. 2, pp. 535-552, Big Sky, Mont, USA, March 2001.

[10] D. Scharf E Hadaegh and B. Kang, "On the validity of the double integrator approximation in deep space formation flying," in Proceedings of the International Symposium on Formation Flying Missions and Technologies, October 2002.

[11] M. S. de Queiroz, V. Kapila, and Q. Yan, "Adaptive nonlinear control of multiple spacecraft formation flying," Journal of Guidance, Control, and Dynamics, vol. 23, no. 3, pp. 385-390, 2000.

[12] H. Peng, J. Zhao, Z. Wu, and W. Zhong, "Optimal periodic controller for formation flying on libration point orbits," Acta Astronautica, vol. 69, no. 7-8, pp. 537-550, 2011.

[13] J. Wang, J. Zhang, X. Cao, and F. Wang, "Optimal satellite formation reconfiguration strategy based on relative orbital elements," Acta Astronautica, vol. 76, pp. 99-114, 2012.

[14] H. Min, S. Wang, F. Sun, Z. Gao, and J. Zhang, "Decentralized adaptive attitude synchronization of spacecraft formation," Systems and Control Letters, vol. 61, no. 1, pp. 238-246, 2012.

[15] J. Wang and Z. Sun, "6-DOF robust adaptive terminal sliding mode control for spacecraft formation flying," Acta Astronautica, vol. 73, pp. 76-87, 2012.

[16] L. Weng, B. Li, W. Cai, R. Zhang, M. J. Zhang, and Y. D. Song, "Human memory/learning inspired approach for attitude control of crew exploration vehicles (CEVs)," in Proceedings of the American Control Conference (ACC '07), pp. 3843-3848, New York, NY, USA, July 2007.

[17] L. Weng and D. Y. Song, "Path planning and path tracking control of Unmanned Ground Vehicles (UGVs)," in Proceedings of the 37th Southeastern Symposium on System Theory (SST05 '05), pp. 262-266, March 2005.

[18] I. Roitt, J. Brostoff, and D. Male, Immunology, 4th edition, 1996. 


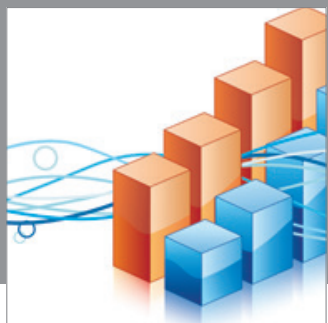

Advances in

Operations Research

mansans

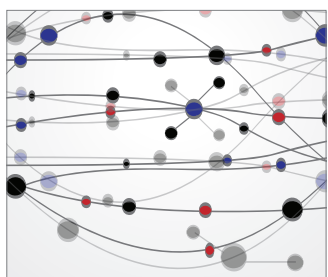

The Scientific World Journal
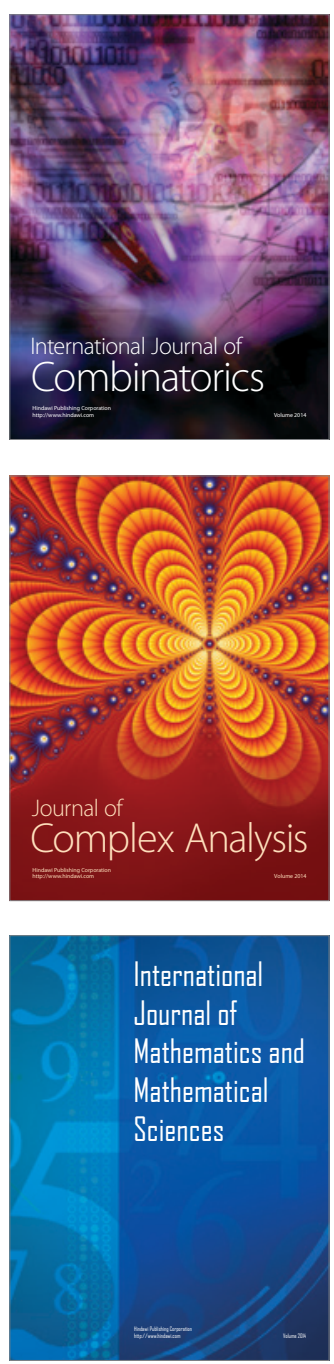
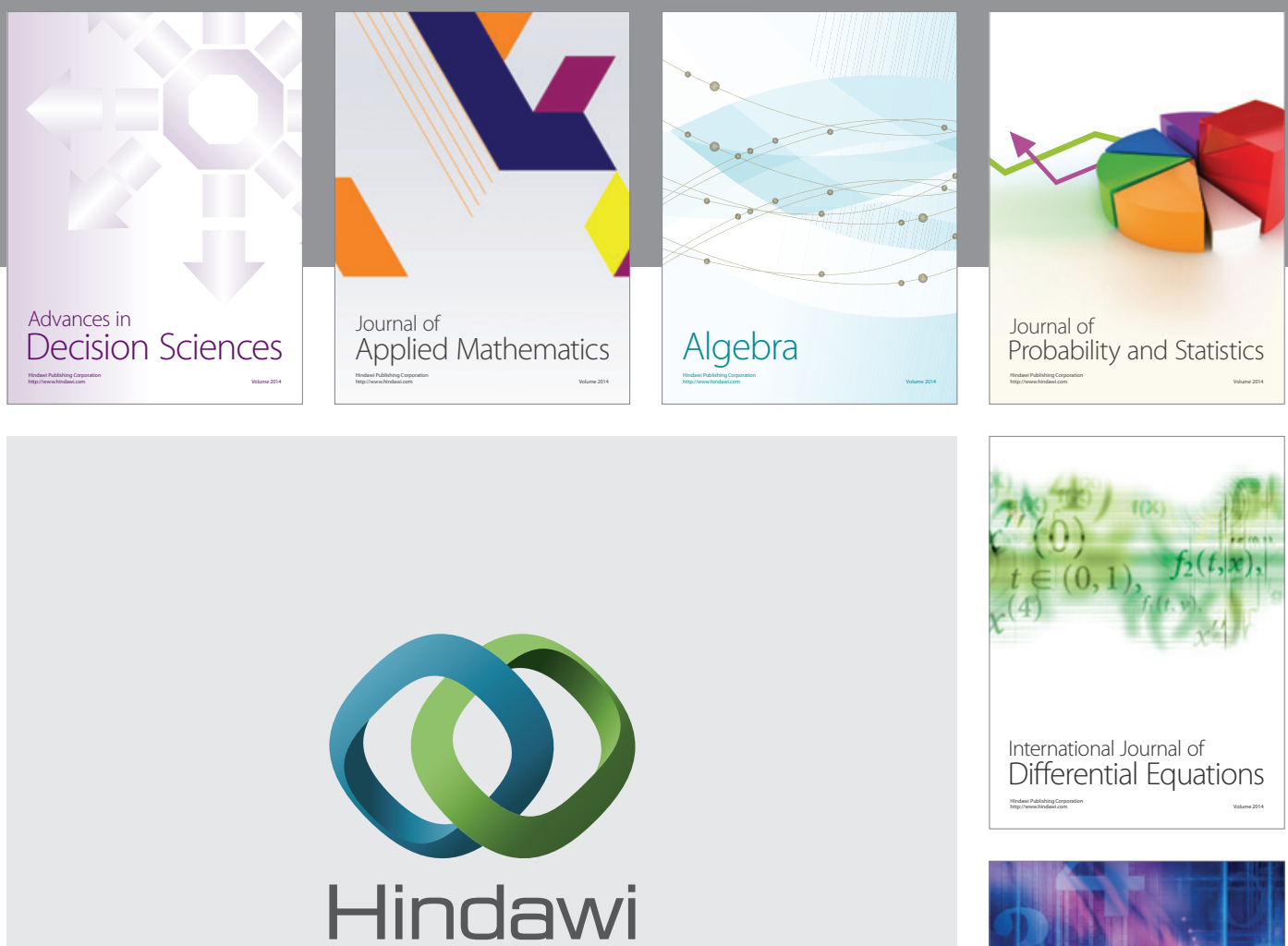

Submit your manuscripts at http://www.hindawi.com
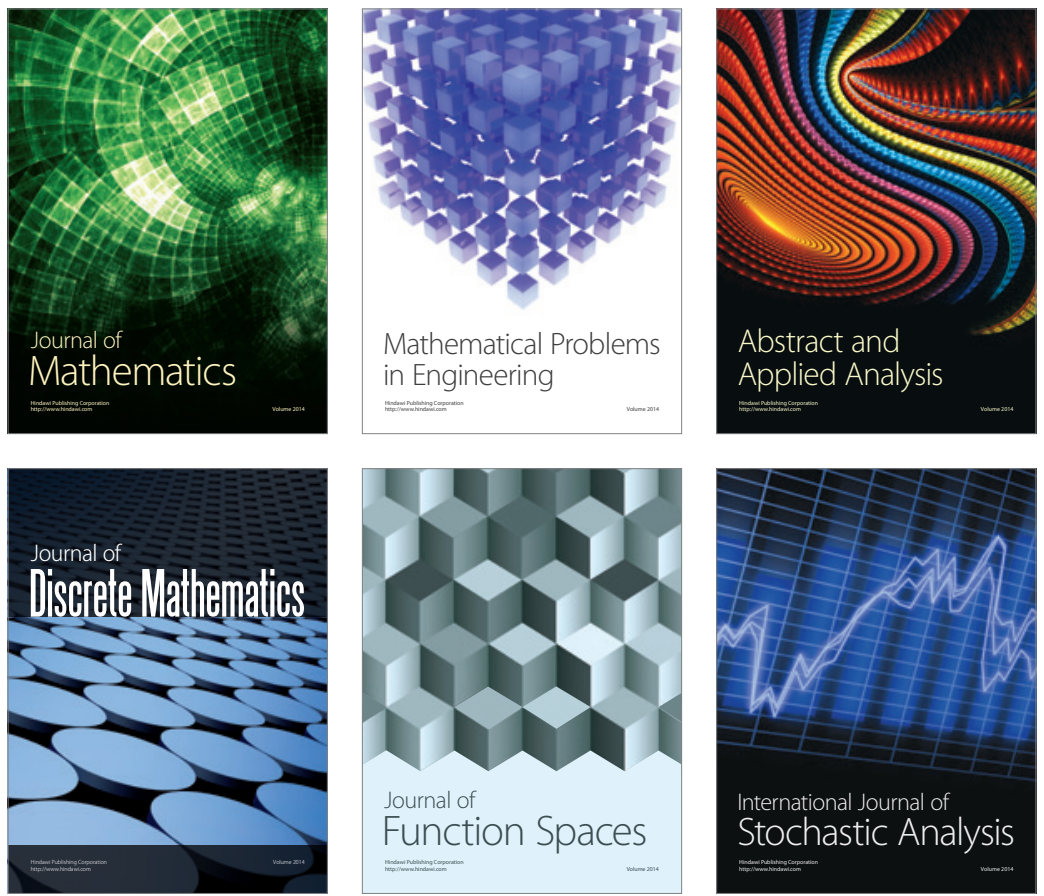

Journal of

Function Spaces

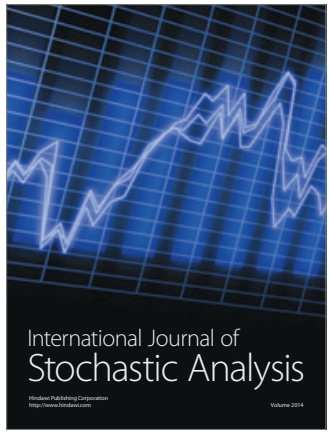

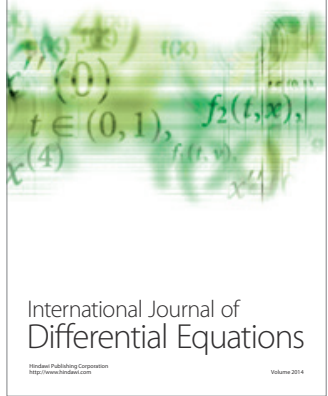
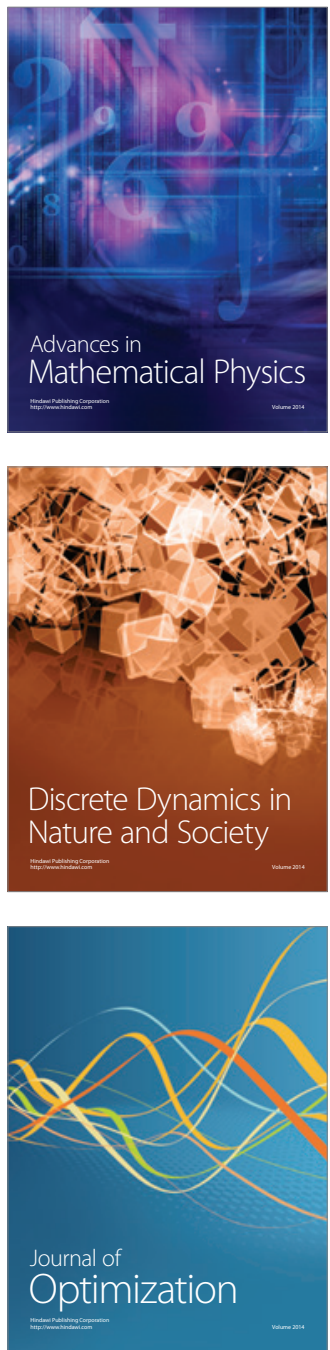